\title{
How and why to diagnose substance use disorders under DSM-5
}

\begin{abstract}
The American Psychiatric Association has been publishing a Diagnostic and Statistical Manual of Mental Disorders since 1952. The fifth edition, published in 2013 after many years of consideration and discussion, contains a major change in thinking about drug and alcohol use. This new thinking of substance abuse and substance dependence as a unified, single entity, diagnosed as a substance use disorder, on a spectrum of mild, moderate and severe. Each specific substance is addressed separately, with consistent criteria for the diagnosis of a substance use disorder, intoxication, withdrawal, or substance-induced disorders. The accurate and consistent use of proper terminology will improve the care of patients, contribute to scholarly discussions and improve the value and validity of research.
\end{abstract}

Keywords: substance use disorder, substance abuse, substance dependence, diagnostic criteria, intoxication, withdrawal
Volume I Issue 2 - 2015

\author{
Philip A Gill \\ Department of Psychiatry and Behavioral Neurosciences, Wayne \\ State University School of Medicine, Detroit, MI, USA
}

Correspondence: Philip A Gilly, MD, Henry Ford Maplegrove Center, 6773 West Maple Road, West Bloomfield, MI, USA, Tel 248-66I-6I00, Fax 248-788-3 I77, Email pgillyl@hfhs.org

Received: June 16,2015 | Published: August 04, 2015
Abbreviations: APA, american psychiatric association; DSM5, diagnostic and statistical manual of mental disorders, fifth edition; SUD, substance use disorder; ICD, international classification of diseases

\section{Introduction}

The American Psychiatric Association (APA) has been publishing a Diagnostic and Statistical Manual of Mental Disorders since 1952. The fifth edition was published in 2013 after many years of consideration and discussion. ${ }^{1}$ It is a lengthy tome and few will have the time or the patience to read it in its entirety. However the chapter on Substance-Related disorders contains many significant differences from the previous edition. Understanding them is critical to the care of patients.

The DSM-5 contains a major change in thinking about drug and alcohol use. Gone are the confusing diagnoses of "abuse" and "dependence". Now these terms can go back to their original function of describing behaviors without trying to also define a symptom complex. The term abuse should be limited to describing either the intentional use of medication for a nonmedical purpose, such as altering one's state of consciousness; or any use of an illegal drug, for example, for the purpose of getting high.

Dependence exists in two forms: Physical dependence is a drug class specific withdrawal syndrome that occurs when there is abrupt cessation, rapid dose reduction, decreasing blood level of the drug, and/or administration of an antagonist.

Psychological dependence is a constellation of substance-induced thoughts and behaviors including craving, compulsion, loss of control, and continued use despite negative consequences.

In common usage, the term "dependence" is invariably used when in actuality what is meant is only physical dependence with or without withdrawal symptoms. Most people associate dependence with "addiction" when in fact physical dependence can be a normal physiologic response to a substance. Tolerance and withdrawal can be normal responses to prescribed medications that affect the nervous system and should be differentiated from addiction. In order to have addiction, a person usually needs to have both physical and psychological dependence.

The fifth edition (DSM-5) asks us instead to think of substance abuse and substance dependence as a unified, single entity, diagnosed as a substance use disorder, on a spectrum of mild, moderate and severe. Each specific substance is addressed separately, with consistent criteria for the diagnosis of a substance use disorder, intoxication, withdrawal, or substance-induced disorders. The Substance-Related Disordersare divided into ten categories of substances:

a. Alcohol

b. Cannabis, (including marijuana, hashish, wax)

c. Caffeine

d. Hallucinogens (including Phencyclidine, Lysergic acid diethylamide, Ecstasy, mushrooms, club, rave drugs)

e. Inhalants

f. Opioids (including opiates, heroin, morphine, methadone, narcotics),

g. Sedatives (muscle relaxers, barbiturates), Hypnotics (sleeping pills), anxiolytics (benzodiazepines)

h. Stimulants (including cocaine, crack, amphetamines, methamphetamine),

i. Tobacco

j. Other (including anabolic steroids) or Unknown substances

Rendering a diagnosis of a Substance Use Disorder (SUD) under DSM-5 requires the person to have a problematic pattern of alcohol or substance use leading to clinically significant impairment or distress as manifested by at least two of a set of 11 criteria, which have occurred during a 12-month period, either presently or in the past. There are only 10 criteria used for hallucinogens or inhalants because they are not considered to generate a specific withdrawal syndrome.

It is preferred to render the diagnosis using the name of the 
specific substance, such as hydrocodone or alprazolam, rather than the substance category, such as opioids or benzodiazepines.

Accurate and efficient information collection is crucial to the rendering of accurate and unbiased diagnoses. ${ }^{2}$ For the sake of consistency, it is preferred to ask the same questions in the same ways of each patient. Including the questions along with their answers within the evaluation documentation that is shared with other clinicians will help to spread the knowledge of how to make fair and impartial diagnoses.

When employing the follow questions, simply replace the word use with whichever substance is relevant: the drinking of alcohol, the use of illicit drugs, the misuse or abuse of prescription, nonprescription medications, or other legal substances.

"Thinking back, is there a 12-month period of time where you could answer YES to any of these questions?

1. Do you find that you use more often, or for a longer time, than you planned for?

2. Do you want to cut back or stop; or have you ever tried and failed to cut back or stop your use?

3. Do you spend a great deal of your time obtaining, taking, or recovering from your use?

4. Do you experience strong desires or cravings to use?

5. Have you repeatedly failed to fulfill major obligations at work, at home, or at school because of your use?

6. Do you continue to use even though you suspect, or even know, that it creates or worsens interpersonal or social problems?

7. Have you given up or reduced important social, occupational, or recreational activities because of your use?

8. Do you repeatedly use in situations in which it is physically hazardous to yourself or others, such as smoking in bed, driving a car, operating a machine, or being at work while intoxicated, impaired, or under the influence?

9. Do you continue to use, even though you suspect, or even know, that it creates or worsens problems with your mind and body?

10. Do you find that you need to use more than in the past in order to achieve the same desired effect, or that you feel less of an effect from the same amount than in the past?

11. *Do you find when you stop or reduce your use, that you experience any withdrawal symptoms, or have you ever taken another substance to prevent or reduce withdrawal symptoms?"

The last criterion Withdrawal does not apply to hallucinogens or inhalants. Another caveat to remember is that the tolerance and withdrawal criteria may not apply when the substance is being prescribed for medical use and is being taken properly.

The complete diagnosis of SUD requires the determination of its Severity and assignment of Specifiers. The levels of severity are determined by the number of criteria that are met. A patient with a SUD of severity Mild will meet two or three criteria; Moderate - four or five criteria; and Severe - six or more criteria.

If the person does not meet any of the criteria, or only one, then they do not yet meet the DSM-5 definition for the diagnosis of a Substance Use Disorder. However, it may be appropriate to think of the person's relationship with drugs or alcohol as being in the "at risk", "problematic", or "hazardous" pattern of use. This will serve the clinician as a reminder to revisit this subject again in the future.

It is appropriate to think of a patient meeting the criteria for a mild substance use disorder as having a relationship with that substance that used to be called "substance abuse." When the patient meets the criteria for either moderate or severe SUD, they should be considered to have what was formerly known as "substance dependence."

This equivalence is important to remember because the current International Classification of Diseases (ICD) diagnosis codes still use the descriptors "abuse" and "dependence". Therefore, even though it is now proper to document diagnoses using DSM-5 verbiage, the assignment of diagnosis codes is still based on the nomenclature from the previous DSM, until the next ICD revision.

There are specifiers that refer to abstinence duration, when no criteria are present other than \#4 Craving: In early remission (more than 3 months but less than 12 months), and In sustained remission (longer than 12 months). During the first 3 months of abstinence, adding "not yet in remission" will provide clarity.

There are also specifiers that denote external influences on abstinence: "On maintenance therapy" if taking opioid substitution or nicotine replacement therapy, and "In a controlled environment" if physically segregated from substance use.

In summary, the DSM-5 has replaced the two categories of substance abuse and substance dependence with a unified, single entity. The diagnosis of substance use disorder now exists on three levels, mild, moderate and severe. Although this is a major departure from long-standing thought and practice, the consistency of criteriadriven diagnoses provides many advantages. The accurate and consistent use of proper terminology will improve the care of our patients, contribute to scholarly discussions and elevate the value and validity of research.

\section{Acknowledgements}

None.

\section{Conflict of interest}

The author declares no conflict of interest.

\section{References}

1. American Psychiatric Association. Diagnostic and statistical manual of mental disorders. 5th ed. Washington, DC, USA: American Psychiatric Publishing; 2013. $991 \mathrm{p}$.

2. Nussbaum AN. The Pocket Guide to the DSM-5 Diagnostic Exam. Washington DC, USA: American Psychiatric Publishing; 2013. p. 129158. 